\title{
Design of Tat Twam Asi-Discrepancy Evaluation Model Based on TOPSIS in Determining the Improvement Priority Aspect
}

\author{
Dewa Gede Hendra Divayana ${ }^{1 *}$, Agus Adiarta ${ }^{2}$, P. Wayan Arta Suyasa ${ }^{3}$ \\ ${ }^{I}$ Department of IT Education,Universitas Pendidikan Ganesha, Jl. Udayana No. 11 Singaraja, Bali, 81116, Indonesia \\ ${ }^{2}$ Department of Electrical Education,Universitas Pendidikan Ganesha, Jl. Udayana No. 11 Singaraja, Bali, 81116, Indonesia \\ ${ }^{3}$ Department of IT Education,Universitas Pendidikan Ganesha, Jl. Udayana No. 11 Singaraja, Bali, 81116, Indonesia
}

\begin{abstract}
This research aimed to provide information about the existence of the new evaluation model in the field of education. The intended evaluation model is called the Tat Twam Asi-Discrepancy evaluation model based on TOPSIS. This model serves to determine the dominant aspects become improvement priority, so later it can trigger the effectiveness of blended learning implementation. The method was used in this research was a development method using the Borg and Gall model, which only focused on the design development stage. Subjects were involved in the initial trials of the evaluation model design were four experts and 30 respondents to conducted simulation trials of TOPSIS calculation. The tools were used to conducted preliminary trials toward the evaluation model design and simulation trials of calculations were questionnaires. Analysis of the initial trial results toward the model design was done by comparing the quality percentage of the test results with the percentage of quality standards that refer to the eleven's scale. Analysis of the simulation test results of the TOPSIS calculation was done by comparing the effectiveness percentage of the test results with the percentage of effectiveness standards that refer to the five's scale. The results of this research indicate this evaluation model is highly qualified and effective is used to determine the most dominant evaluation aspect to become an improvement priority to realize the effectiveness of blended learning implementation.
\end{abstract}

Keywords:

Evaluation Model;

Tat Twam Asi;

Discrepancy;

TOPSIS;

Blended Learning.

\begin{tabular}{llll}
\multicolumn{2}{l}{ Article History: } & & \\
Received: & 02 & February & 2021 \\
Revised: & 09 & May & 2021 \\
Accepted: & 22 & May & 2021 \\
Published: & 01 & June & 2021
\end{tabular}

\section{1- Introduction}

The learning process in schools has changed since the appearance of the Industry Revolution 4.0 era. One of those changes is the change in learning models applied in schools. In general, most senior high schools or vocational schools in Indonesia have implemented blended learning as a learning model that is used in the learning process [1]. Nevertheless, the facts in the field show that there are still several senior high schools or vocational schools of IT (especially in Bali) that have not been fully effective in implementing blended learning as a learning model [2]. The ineffectiveness is caused by several aspects, included: the unpreparedness legality of the blended learning implementation, the unpreparedness of academic community support in organizing blended learning, the unpreparedness of budgetary, the unpreparedness of the development team's ability, the unpreparedness of the users' capability to operate the blended learning, the unpreparedness of supporting infrastructures, and so on. From those aspects, it is necessary to know the dominant aspect as a priority to be improved so that from the beginning can is conducted optimal efforts to realize the effectiveness of blended learning implementation.

*CONTACT: Hendra.divayana@undiksha.ac.id

DOI: http://dx.doi.org/10.28991/esj-2021-01285

(C) 2021 by the authors. Licensee ESJ, Italy. This is an open access article under the terms and conditions of the Creative Commons Attribution (CC-BY) license (https://creativecommons.org/licenses/by/4.0/). 
Based on those problems, it is necessary to do a holistic evaluation of the blended learning implementation at senior high schools or vocational schools of IT in Bali. Some of the efforts that had been conducted were evaluation activities using several general models of educational evaluation, included: CIPP, Countenance, CSE-UCLA, Discrepancy, and others $[3,4]$. However, some of those general models have not been able to holistically and accurately determine the dominant aspect that is the priority of improvement. Based on that situation, it is necessary to modify those general models of educational evaluation.

One effort that can be done is to modify the Discrepancy model with the Tat Twam Asi concept and the TOPSIS method. The Discrepancy model is an educational evaluation model that shows the imbalances or differences that occur between the evaluation results in the field with established evaluation standards [5]. The Discrepancy model consists of four evaluation components, included: definition, installation, process, and product [6]. Tat Twam Asi is the concept of local wisdom in Bali that upholds equality rights in social relationships to maintain harmony in life [7]. Tat Twam Asi is also often interpreted with the phrase "I am you" or "I and you are the same". That phrase implies equality [8].

The TOPSIS (Technique for Order Preference by Similarity to Ideal Solution) method is one of the decision support system methods that the principle of finding choice alternatives by determining the farthest distance from negative ideal solutions and the shortest distance from positive ideal solutions. The negative ideal solutions consist of all the worst values that can be achieved by criteria, while, the positive ideal solutions consist of all the best values that can be achieved by criteria [9, 10]. Referring to that efforts, so this research question: "how is the design of the Discrepancy model modified with the Tat Twam Asi concept and the TOPSIS method in determining the dominant aspects, that need to be improved to trigger the effectiveness of blended learning implementation, especially at IT vocational schools in Bali?"

This research was base-lined on some studies that had been conducted previously by several researchers regarding the evaluation of the blended learning implementation or distance education. The research was conducted by Mutawa (2017) [11] showed that the evaluation of blended learning used the Technology Acceptance Module (TAM), but did not use an educational evaluation model specifically. The limitation of Mutawa's research showed there were no aspects of educational evaluation that were used as a basis for evaluating blended learning in the view of educational dimensions. The research was conducted by Lippe and Carter (2018) [12] showed an evaluation of the distance learning model using the CIPP model. The limitation of Lippe and Carter's research was it had not shown the dominant aspect become improvement priority, so it was challenging to implement all the recommendations that were recommended simultaneously at the same time. The research was conducted by Thurab-Nkhosi (2019) [13] showed the use of the CIPP model to evaluate blended learning that was implemented at the faculty level. The limitation of Thurab-Nkhosi's research was it had not shown the complete presentation of evaluation results in each of the evaluation components of context, input, process, and product. Besides, Thurab-Nkhosi's research also was it had not shown the calculation process in determining the dominant aspect, which was prioritized for improvement to realize the effectiveness of blended learning implementation.

The research was conducted by Martín-Martínez et al. (2020) [14] showed the existence of evaluation activities toward blended learning. The limitation of Martín-Martínez et al.'s research was that it had not shown the dominant aspect that becomes improvement priority. The research was conducted by Mantara et al. (2020) [15] showed evaluation activities toward learning media used in online learning during the Covid-19 pandemic. The limitation of Mantara et al.'s research was it had not shown the educational evaluation model used in evaluating the learning media in online learning. The research was conducted by Habib and Ramzan (2020) [16] showed the results of performance assessment and analysis of blended learning. The limitation of Habib and Ramzan's research was it had not shown a specific educational evaluation model to be used as a basis for conducting the assessment. The research was conducted by Naibaho (2021) [17] showed evaluation activity toward online learning using the CSE-UCLA evaluation model. The limitation of Naibaho's research was that it had not shown the dominant aspect that needs to be improved for enhancing online learning.

\section{2- Material and Methods}

This research used a development approach. The development method was used refers to the Borg and Gall development design, which focuses on the design development stage. At the design development stage, several attributes were needed, included: evaluation aspects of the Discrepancy model, the percentage of evaluation success standards, the imbalances values of each evaluation aspect, weights of decision-makers refer to the Tat Twam Asi concept, and the TOPSIS formula.

The numbers of experts were involved in conducting the initial trials of the evaluation model design were four experts (two education experts and two informatics experts). In addition to experts, 30 teachers were involved in conducting a simulation of TOPSIS calculation to determine the dominant aspect that needed to be improved. Those teachers came from several IT vocational schools in six regencies in Bali, included: Tabanan, Klungkung, Denpasar, Badung, Buleleng, and Gianyar. 
The tools that were used to collect data of the initial trial results toward the evaluation model design used questionnaires, each consisting of 10 questions with a choice of answers from each question based on the Likert measurement scale. The tools were used to collect data of the results of TOPSIS calculation simulation trials used questionnaires were consisting of 10 questions with the choice of answers to each question, also referring to the Likert measurement scale.

The data analysis from the initial trial results of the evaluation model design was done by comparing the average of the quality percentage of the design trial results with the percentage of the design quality standard that refers to the eleven's scale. The quality standards of eleven's scale consist of scores range of 95-100 for the 'Excellent' category, scores of 85-94 for the 'Good' category, scores of 75-84 for the 'Advanced' category, scores of 65-74 for the 'Intermediate' category, scores of 55-64 for the 'Enough' category, scores of 45-54 for the 'Elementary' category, scores of 35-44 for the 'Less' category, scores of 25-34 for the 'Very Less' category, scores of 15-24 for the 'Bad' category, scores of 5-14 for the 'Very Bad' category, and scores of 0-4 for the 'Poor' category [18]. If the quality standards percentage of the design trial results that have been obtained include in the excellent category, good, advanced, and intermediate, then there is no need to revise the evaluation model design. If outside the category, then it needs to conduct a re-trial of the evaluation model design by involving more respondents and have a deeper perspective on the evaluation model design.

Analysis of the simulation trial results of the TOPSIS calculation was done by comparing the effectiveness percentage average of the calculation trial results with the standard percentage of calculation effectiveness that refers to the five's scale. The effectiveness standards of five's scale consist of scores range of 90-100 for the 'Very Effective' category, scores of 80-89 for the 'Effective' category, scores of 65-79 for the 'Moderate' category, scores of 55-64 for the 'Less Effective' category, and scores of 0-54 for the 'Ineffective' category [19, 20]. If the percentage of calculation effectiveness standards that have been obtained include in a very effective and effective category, then there is no need to revise/re-calculate the TOPSIS calculation. If outside the category, then it necessary to re-simulate the TOPSIS calculation by involving more qualified respondents.

The formula that is used to determine the percentage of quality standards and effectiveness standards [21] can be seen in Equation 1. The formula for simulation of the TOPSIS calculation consists of several equations, included: Equation 2 for calculating normalized matrix [22], Equation 3 for determining scores of matrix-Y [23], Equations 4 and 5 for determining the matrix of negative ideal solutions $\left(\mathrm{A}^{-}\right)$and matrix of positive ideal solutions $\left(\mathrm{A}^{+}\right)$[24], Equations 6 and 7 to determine the distance between the values of each indicator with the negative ideal solutions $\left(\mathrm{D}_{\mathrm{i}}^{-}\right)$ and the positive ideal solutions $\left(\mathrm{D}_{\mathrm{i}}^{+}\right)[25,26]$, and Equation 8 to determine the preference scores of each indicator $\left(\mathrm{V}_{\mathrm{i}}\right)$ [27].

$\mathrm{P}=(\mathrm{f} / \mathrm{N}) \times 100 \%$

where: P: percentage of quality or effectiveness; $\mathrm{f}$ : the total scores that have been obtained; and $\mathrm{N}$ : the total of maximum scores.

$$
r_{i j}=\frac{x_{i j}}{\sqrt{\sum_{i=1}^{m} x_{i j}^{2}}}
$$

where: $i=1,2,3, \ldots . m$, and $j=1,2,3 \ldots n$; $x_{i j}$ : basic matrix; $i$ : the row of the matrix; $j$ : the column of the matrix; and $r_{i j}$ : matrix of normalized results from the basic matrix.

$\mathrm{y}_{\mathrm{ij}}=\mathrm{w}_{\mathrm{i} * \mathrm{r}_{\mathrm{ij}}}$

where: $\mathrm{y}_{\mathrm{ij}}$ : Matrix-Y; $\mathrm{w}_{\mathrm{i}}$ : the weights of the decision-makers (the weights that have been generalized by using the concept of Tat Twam Asi); and $\mathrm{r}_{\mathrm{ij}}$ : Matrix-R.

$A^{-}=\left(y_{1}^{-}, y_{2}^{-}, \cdots, y_{n}^{-}\right)$

$A^{+}=\left(y_{1}^{+}, y_{2}^{+}, \cdots, y_{n}^{+}\right)$

where:

$y_{j}^{-}= \begin{cases}\min _{i} y_{i j} ; & \text { if } \mathrm{j} \text { is benefit attribute } \\ \max _{i} y_{i j} ; & \text { if } \mathrm{j} \text { is cost attribute }\end{cases}$
$y_{j}^{+}= \begin{cases}\max _{i} y_{i j} ; & \text { if } \mathrm{j} \text { is benefit attribute } \\ \min _{i} y_{i j} ; & \text { if } \mathrm{j} \text { is cost attribute }\end{cases}$ 


$$
\begin{aligned}
D_{i}^{-} & =\sqrt{\sum_{j=1}^{n}\left(y_{i j}-y_{i}^{-}\right)^{2}} \\
D_{i}^{+} & =\sqrt{\sum_{j=1}^{n}\left(y_{i}^{+}-y_{i j}\right)^{2}} \\
V_{i} & =\frac{D_{i}^{-}}{D_{i}^{-}+D_{i}^{+}}
\end{aligned}
$$

\section{3- Results and Discussion}

This research had succeeded in obtaining data related to the five essential components that were used as a basis for designing the Discrepancy evaluation model that modification by the Tat Twam Asi concept and the TOPSIS method. Those components, included: 1) the evaluation aspects of the Discrepancy model, 2) the percentage of evaluation success standards, 3) the imbalance values of each evaluation aspect, 4) the weights of the decision-makers that refers to the Tat Twam Asi concept, and 5) the TOPSIS formula. The evaluation aspects of the Discrepancy model completely can be seen in Table 1. The percentage of evaluation success standards can be seen in Table 2 . The imbalance values of each evaluation aspect can be seen in Table 3. The weights of the decision-makers that refer to the Tat Twam Asi concept can be seen in Table 4. The TOPSIS formula has been explained previously in Equations 2 to 8 .

\begin{tabular}{|c|c|c|}
\hline Evaluation Components & & Evaluation Aspects \\
\hline \multirow{3}{*}{ Definition } & A1 & Legal regulations for the implementation of blended learning \\
\hline & $\mathrm{A} 2$ & Parents' support \\
\hline & A3 & Teachers and students' support \\
\hline \multirow{4}{*}{ Installation } & A4 & Funding Readiness \\
\hline & A5 & The readiness of facilities and infrastructures \\
\hline & A6 & Management team readiness \\
\hline & A7 & The preparedness of users' ability \\
\hline \multirow{3}{*}{ Process } & A8 & Socialization about the use of blended learning \\
\hline & A9 & Implementation of learning through blended learning \\
\hline & A10 & Funding management \\
\hline \multirow{5}{*}{ Product } & A11 & Users' satisfaction on the tangible dimension \\
\hline & A12 & Users' satisfaction on the reliability dimension \\
\hline & A13 & Users' satisfaction on the responsiveness dimension \\
\hline & A14 & Users' satisfaction on the assurance dimension \\
\hline & A15 & Users' satisfaction on the empathy dimension \\
\hline
\end{tabular}

Table 1. Evaluation aspects of the Discrepancy model that was used to evaluate the blended learning implementation at IT vocational schools in Bali.

Table 2. Percentage of success standards for evaluating the blended learning implementation at IT vocational schools in Bali.

\begin{tabular}{cc}
\hline Codes of aspects & The percentage of evaluation success standards \\
\hline A1 & $\geq 88 \%$ \\
A2 & $\geq 88 \%$ \\
A3 & $\geq 88 \%$ \\
A4 & $\geq 82 \%$ \\
A5 & $\geq 82 \%$ \\
A6 & $\geq 85 \%$ \\
A7 & $\geq 84 \%$ \\
A8 & $\geq 85 \%$ \\
A9 & $\geq 84 \%$ \\
A10 & $\geq 90 \%$ \\
A11 & $\geq 85 \%$ \\
A12 & $\geq 85 \%$ \\
A13 & $\geq 85 \%$ \\
A14 & $\geq 85 \%$ \\
A15 & $\geq 85 \%$ \\
\hline
\end{tabular}


Table 3. Imbalance values for each aspect of evaluation.

\begin{tabular}{cccc}
\hline Codes of aspects & $\begin{array}{c}\text { Percentage of assessment from } \\
\text { 30 respondents (\%) }\end{array}$ & $\begin{array}{c}\text { Minimum percentage of the } \\
\text { evaluation success standards (\%) }\end{array}$ & Imbalance Values (\%) \\
\hline A1 & 91.333 & 88.000 & 3.333 \\
A2 & 90.667 & 88.000 & 2.667 \\
A3 & 88.667 & 88.000 & 0.667 \\
A4 & 79.333 & 82.000 & -2.667 \\
A5 & 80.000 & 82.000 & -2.000 \\
A6 & 87.333 & 85.000 & 2.333 \\
A7 & 88.667 & 84.000 & 4.667 \\
A8 & 88.667 & 85.000 & 3.667 \\
A9 & 88.000 & 84.000 & 4.000 \\
A10 & 76.667 & 90.000 & -13.333 \\
A11 & 88.000 & 85.000 & 3.000 \\
A12 & 89.333 & 85.000 & 4.333 \\
A13 & 88.667 & 85.000 & 3.667 \\
A14 & 88.667 & 85.000 & 3.667 \\
A15 & 87.333 & 85.000 & 2.333 \\
\hline & & & \\
\hline
\end{tabular}

Table 4. The weights of the experts/decision-makers that refers to the Tat Twam Asi concept.

\begin{tabular}{|c|c|c|c|c|c|c|}
\hline \multirow{2}{*}{ Aspects } & \multicolumn{4}{|c|}{ Weights } & \multirow{2}{*}{$\begin{array}{c}\text { Average of } \\
\text { weights }\end{array}$} & \multirow{2}{*}{$\begin{array}{c}\text { Tat Twam Asi-Based } \\
\text { Weights }\end{array}$} \\
\hline & Expert-1 & Expert-2 & Expert-3 & Expert-4 & & \\
\hline A1 & 5 & 4 & 5 & 5 & 4.750 & 0.071 \\
\hline $\mathrm{A} 2$ & 4 & 4 & 4 & 4 & 4.000 & 0.060 \\
\hline A3 & 4 & 4 & 4 & 4 & 4.000 & 0.060 \\
\hline A4 & 4 & 4 & 3 & 4 & 3.750 & 0.056 \\
\hline A5 & 4 & 4 & 4 & 3 & 3.750 & 0.056 \\
\hline A6 & 4 & 5 & 4 & 5 & 4.500 & 0.067 \\
\hline A7 & 4 & 4 & 5 & 3 & 4.000 & 0.060 \\
\hline A8 & 4 & 4 & 5 & 5 & 4.500 & 0.067 \\
\hline A9 & 4 & 5 & 4 & 4 & 4.250 & 0.063 \\
\hline $\mathrm{A} 10$ & 4 & 5 & 5 & 5 & 4.750 & 0.071 \\
\hline A11 & 4 & 5 & 5 & 4 & 4.500 & 0.067 \\
\hline A 12 & 4 & 5 & 4 & 5 & 4.500 & 0.067 \\
\hline A13 & 4 & 5 & 4 & 5 & 4.500 & 0.067 \\
\hline A14 & 5 & 4 & 4 & 5 & 4.500 & 0.067 \\
\hline A15 & 4 & 5 & 4 & 5 & 4.500 & 0.067 \\
\hline A16 & 2 & 2 & 2 & 3 & 2.250 & 0.034 \\
\hline$\Sigma$ & & & & & 67.000 & 1.000 \\
\hline
\end{tabular}

Design of Discrepancy evaluation model that modification with the concept of Tat Twam Asi and the TOPSIS method was able to be made after the attributes of the design completeness were fulfilled. That model design can be seen in Figure 1. 


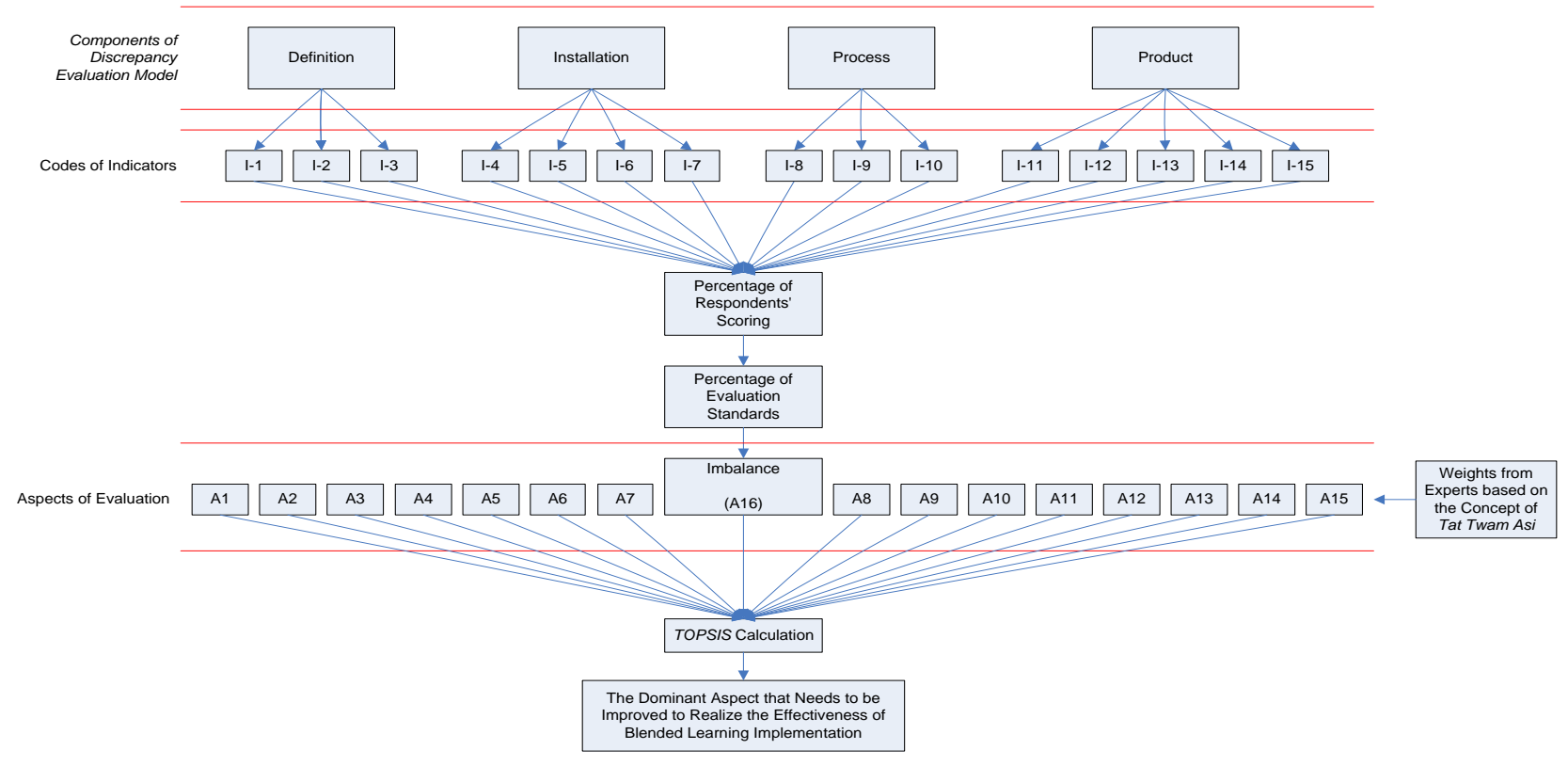

Figure 1. Design of Discrepancy evaluation model that modified by the Tat Twam Asi and TOPSIS.

Figure 1 above showed the evaluation model design made from the combination of the Discrepancy model, the Tat Twam Asi concept, and the TOPSIS method. The Discrepancy model consists of four components, included: Definition, Installation, Process, and Product. The definition component consists of three indicators that contain questions related to the definition component. Likewise, the installation component consists of four indicators. The process component consists of three indicators, and the product component consists of five indicators. The respondents gave rating scores for those indicators. Respondent's rating scores for each indicator were converted into percentages form. The percentage of respondents' assessment then compared with the minimum percentage of the evaluation success standards. The results of that comparison showed imbalance values. That imbalance was used as the $16^{\text {th }}$ aspect (an aspect of imbalance) of a total of 15 aspects of the Discrepancy evaluation model that had existed previously. That sixteen evaluation aspects were given equal weights from experts who refer to the Tat Twam Asi concept, and then a TOPSIS calculation was performed. The TOPSIS calculation results were used as a determinant of the dominant aspect that needs to be improved to realize the effectiveness of blended learning implementation.

Initial trials toward the design of the Tat Twam Asi-Discrepancy evaluation model based on TOPSIS were conducted to determine the percentage of the design quality. The initial trial results that had been carried out by four experts on the evaluation model design can be seen in Table 5.

Table 5. Initial trial results toward the design of the Tat Twam Asi-Discrepancy evaluation model based on TOPSIS.

\begin{tabular}{|c|c|c|c|c|c|c|c|c|c|c|c|c|c|}
\hline \multirow{2}{*}{ No. } & \multirow{2}{*}{ Respondents } & \multicolumn{10}{|c|}{ Items- } & \multirow{2}{*}{$\sum$} & \multirow{2}{*}{ Quality Percentage (\%) } \\
\hline & & 1 & 2 & 3 & 4 & 5 & 6 & 7 & 8 & 9 & 10 & & \\
\hline 1 & Education Expert-1 & 5 & 4 & 4 & 5 & 4 & 4 & 4 & 5 & 4 & 5 & 44 & 88.00 \\
\hline 2 & Education Expert-2 & 4 & 4 & 4 & 4 & 5 & 4 & 5 & 4 & 4 & 4 & 42 & 84.00 \\
\hline 3 & Informatics Expert-1 & 5 & 4 & 5 & 4 & 4 & 4 & 4 & 4 & 4 & 5 & 43 & 86.00 \\
\hline 4 & Informatics Expert-2 & 4 & 5 & 4 & 4 & 5 & 4 & 5 & 4 & 5 & 5 & 45 & 90.00 \\
\hline \multicolumn{13}{|c|}{ Average } & 87.00 \\
\hline
\end{tabular}

Notes:

Item-1: Completeness of the evaluation components;

Item-2: Suitable instrument items for each evaluation component;

Item-3: Clarity of the respondent's assessment component of each instrument item in each evaluation aspect;

Item-4: Clarity of minimum standards for evaluation success;

Item-5: Suitable aspects with the Discrepancy model evaluation;

Item-6: Suitable aspects of imbalance;

Item-7: Completeness of the expert's weights;

Item-8: Clarity of TOPSIS calculation components;

Item-9: Clarity of the stages to determine the dominant aspects;

Item-10: Appropriate data flow chart in the design. 
Based on the data shown in Table 3, initial data was able to be compiled to determine the normalized matrix needed in the TOPSIS calculation process. That initial data can be seen in Table 6.

Table 6. Preliminary data for normalized matrix calculations.

\begin{tabular}{|c|c|c|c|c|c|c|c|c|c|c|c|c|c|c|c|c|}
\hline \multirow{2}{*}{ Indicators } & \multicolumn{16}{|c|}{ Aspects } \\
\hline & A1 & A2 & $\mathbf{A 3}$ & A4 & A5 & A6 & A7 & A8 & A9 & A10 & A11 & A12 & A13 & A14 & A15 & A16 \\
\hline I-1 & 91.333 & 86.756 & 86.756 & 86.756 & 86.756 & 86.756 & 86.756 & 86.756 & 86.756 & 86.756 & 86.756 & 86.756 & 86.756 & 86.756 & 86.756 & 3.333 \\
\hline $\mathrm{I}-2$ & 86.756 & 90.667 & 86.756 & 86.756 & 86.756 & 86.756 & 86.756 & 86.756 & 86.756 & 86.756 & 86.756 & 86.756 & 86.756 & 86.756 & 86.756 & 2.667 \\
\hline $\mathrm{I}-3$ & 86.756 & 86.756 & 88.667 & 86.756 & 86.756 & 86.756 & 86.756 & 86.756 & 86.756 & 86.756 & 86.756 & 86.756 & 86.756 & 86.756 & 86.756 & 0.667 \\
\hline $\mathrm{I}-4$ & 86.756 & 86.756 & 86.756 & 79.333 & 86.756 & 86.756 & 86.756 & 86.756 & 86.756 & 86.756 & 86.756 & 86.756 & 86.756 & 86.756 & 86.756 & -2.667 \\
\hline $\mathrm{I}-5$ & 86.756 & 86.756 & 86.756 & 86.756 & 80.000 & 86.756 & 86.756 & 86.756 & 86.756 & 86.756 & 86.756 & 86.756 & 86.756 & 86.756 & 86.756 & -2.000 \\
\hline I-6 & 86.756 & 86.756 & 86.756 & 86.756 & 86.756 & 87.333 & 86.756 & 86.756 & 86.756 & 86.756 & 86.756 & 86.756 & 86.756 & 86.756 & 86.756 & 2.333 \\
\hline $\mathrm{I}-7$ & 86.756 & 86.756 & 86.756 & 86.756 & 86.756 & 86.756 & 88.667 & 86.756 & 86.756 & 86.756 & 86.756 & 86.756 & 86.756 & 86.756 & 86.756 & 4.667 \\
\hline $\mathrm{I}-8$ & 86.756 & 86.756 & 86.756 & 86.756 & 86.756 & 86.756 & 86.756 & 88.667 & 86.756 & 86.756 & 86.756 & 86.756 & 86.756 & 86.756 & 86.756 & 3.667 \\
\hline I-9 & 86.756 & 86.756 & 86.756 & 86.756 & 86.756 & 86.756 & 86.756 & 86.756 & 88.000 & 86.756 & 86.756 & 86.756 & 86.756 & 86.756 & 86.756 & 4.000 \\
\hline $\mathrm{I}-10$ & 86.756 & 86.756 & 86.756 & 86.756 & 86.756 & 86.756 & 86.756 & 86.756 & 86.756 & 76.667 & 86.756 & 86.756 & 86.756 & 86.756 & 86.756 & -13.333 \\
\hline I-11 & 86.756 & 86.756 & 86.756 & 86.756 & 86.756 & 86.756 & 86.756 & 86.756 & 86.756 & 86.756 & 88.000 & 86.756 & 86.756 & 86.756 & 86.756 & 3.000 \\
\hline I-12 & 86.756 & 86.756 & 86.756 & 86.756 & 86.756 & 86.756 & 86.756 & 86.756 & 86.756 & 86.756 & 86.756 & 89.333 & 86.756 & 86.756 & 86.756 & 4.333 \\
\hline I-13 & 86.756 & 86.756 & 86.756 & 86.756 & 86.756 & 86.756 & 86.756 & 86.756 & 86.756 & 86.756 & 86.756 & 86.756 & 88.667 & 86.756 & 86.756 & 3.667 \\
\hline I-14 & 86.756 & 86.756 & 86.756 & 86.756 & 86.756 & 86.756 & 86.756 & 86.756 & 86.756 & 86.756 & 86.756 & 86.756 & 86.756 & 88.667 & 86.756 & 3.667 \\
\hline I-15 & 86.756 & 86.756 & 86.756 & 86.756 & 86.756 & 86.756 & 86.756 & 86.756 & 86.756 & 86.756 & 86.756 & 86.756 & 86.756 & 86.756 & 87.333 & 2.333 \\
\hline
\end{tabular}

Notes:

- Orange blocked data were obtained from the assessment percentages of 30 respondents for each aspect that had been shown previously in Table 3.

- Yellow blocked data were obtained from the percentage of imbalance values that had been shown previously in Table 3.

- Unblocked data were obtained from the average of the assessment percentage of 30 respondents that had been shown previously in Table 3.

Based on the data in Table 6, the normalized matrix calculations were able to be performed used Equation 2. The calculation results of the normalized matrix can be seen as follows:

\begin{tabular}{|c|c|c|c|c|c|c|c|c|c|}
\hline $\mathrm{r}_{11}$ & $=0.2708$ & $r_{44}$ & $=0.2374$ & $\mathrm{r}_{77}$ & $=0.2635$ & $r_{1010}$ & $=0.2299$ & $r_{1313}$ & $=0.2635$ \\
\hline$r_{21}$ & $=0.2573$ & $\mathrm{r}_{54}$ & $=0.2596$ & $\mathrm{r}_{87}$ & $=0.2578$ & $\mathrm{r}_{1110}$ & $=0.2601$ & $r_{1413}$ & $=0.2578$ \\
\hline$r_{31}$ & $=0.2573$ & $r_{64}$ & $=0.2596$ & r97 & $=0.2578$ & $\mathrm{r}_{1210}$ & $=0.2601$ & $\mathrm{r}_{1513}$ & $=0.2578$ \\
\hline$r_{41}$ & $=0.2573$ & $\mathrm{r}_{74}$ & $=0.2596$ & $\mathrm{r}_{107}$ & $=0.2578$ & $r_{1310}$ & $=0.2601$ & $\mathrm{r}_{114}$ & $=0.2578$ \\
\hline$r_{51}$ & $=0.2573$ & $\mathrm{r}_{84}$ & $=0.2596$ & $\mathrm{r}_{117}$ & $=0.2578$ & $\mathrm{r}_{1410}$ & $=0.2601$ & $\mathrm{r}_{214}$ & $=0.2578$ \\
\hline$r_{61}$ & $=0.2573$ & $\mathrm{r}_{94}$ & $=0.2596$ & $r_{127}$ & $=0.2578$ & $\mathrm{r}_{1510}$ & $=0.2601$ & $r_{314}$ & $=0.2578$ \\
\hline$r_{71}$ & $=0.2573$ & $\mathrm{r}_{104}$ & $=0.2596$ & $r_{137}$ & $=0.2578$ & $\mathrm{r}_{111}$ & $=0.2580$ & $\mathrm{r}_{414}$ & $=0.2578$ \\
\hline$r_{81}$ & $=0.2573$ & $\mathrm{r}_{114}$ & $=0.2596$ & $r_{147}$ & $=0.2578$ & $\mathrm{r}_{211}$ & $=0.2580$ & $\mathrm{r}_{514}$ & $=0.2578$ \\
\hline $\mathrm{r}_{91}$ & $=0.2573$ & $\mathrm{r}_{124}$ & $=0.2596$ & $\mathrm{r}_{157}$ & $=0.2578$ & $\mathrm{r}_{311}$ & $=0.2580$ & $\mathrm{r}_{614}$ & $=0.2578$ \\
\hline$r_{101}$ & $=0.2573$ & $r_{134}$ & $=0.2596$ & $\mathrm{r}_{18}$ & $=0.2578$ & $r_{411}$ & $=0.2580$ & $\mathrm{r}_{714}$ & $=0.2578$ \\
\hline $\mathrm{r}_{111}$ & $=0.2573$ & $r_{144}$ & $=0.2596$ & $\mathrm{r}_{28}$ & $=0.2578$ & $r_{511}$ & $=0.2580$ & r814 & $=0.2578$ \\
\hline$r_{121}$ & $=0.2573$ & $\mathrm{r}_{154}$ & $=0.2596$ & $\mathrm{r}_{38}$ & $=0.2578$ & $\mathrm{r}_{611}$ & $=0.2580$ & $\mathrm{r}_{914}$ & $=0.2578$ \\
\hline$r_{131}$ & $=0.2573$ & $\mathrm{r}_{15}$ & $=0.2595$ & $\mathrm{r}_{48}$ & $=0.2578$ & $\mathrm{r}_{711}$ & $=0.2580$ & $r_{1014}$ & $=0.2578$ \\
\hline$r_{141}$ & $=0.2573$ & $\mathrm{r}_{25}$ & $=0.2595$ & $\mathrm{r}_{58}$ & $=0.2578$ & $\mathrm{r} 811$ & $=0.2580$ & $\mathrm{r}_{1114}$ & $=0.2578$ \\
\hline$r_{151}$ & $=0.2573$ & $\mathrm{r}_{35}$ & $=0.2595$ & $\mathrm{r}_{68}$ & $=0.2578$ & r911 & $=0.2580$ & $\mathrm{r}_{1214}$ & $=0.2578$ \\
\hline$r_{12}$ & $=0.2574$ & $\mathrm{r}_{45}$ & $=0.2595$ & $\mathrm{r}_{78}$ & $=0.2578$ & $\mathrm{r}_{1011}$ & $=0.2580$ & $\mathrm{r}_{1314}$ & $=0.2578$ \\
\hline $\mathrm{r}_{22}$ & $=0.2690$ & $r_{55}$ & $=0.2393$ & $\mathrm{r} 88$ & $=0.2635$ & $\mathrm{r}_{1111}$ & $=0.2617$ & $\mathrm{r}_{1414}$ & $=0.2635$ \\
\hline$r_{32}$ & $=0.2574$ & $r_{65}$ & $=0.2595$ & $r_{98}$ & $=0.2578$ & $\mathrm{r}_{1211}$ & $=0.2580$ & $\mathrm{r}_{1514}$ & $=0.2578$ \\
\hline$r_{42}$ & $=0.2574$ & $\mathrm{r}_{75}$ & $=0.2595$ & $r_{108}$ & $=0.2578$ & $\mathrm{r}_{1311}$ & $=0.2580$ & $\mathrm{r}_{115}$ & $=0.2581$ \\
\hline$r_{52}$ & $=0.2574$ & $\mathrm{r}_{85}$ & $=0.2595$ & $\mathrm{r}_{118}$ & $=0.2578$ & $\mathrm{r}_{1411}$ & $=0.2580$ & $\mathrm{r}_{215}$ & $=0.2581$ \\
\hline$r_{62}$ & $=0.2574$ & $\mathrm{r}_{95}$ & $=0.2595$ & $\mathrm{r}_{128}$ & $=0.2578$ & $r_{1511}$ & $=0.2580$ & $r_{315}$ & $=0.2581$ \\
\hline$r_{72}$ & $=0.2574$ & $\mathrm{r}_{105}$ & $=0.2595$ & $\mathrm{r}_{138}$ & $=0.2578$ & $\mathrm{r}_{112}$ & $=0.2577$ & $\mathrm{r}_{415}$ & $=0.2581$ \\
\hline$r_{82}$ & $=0.2574$ & $\mathrm{r}_{115}$ & $=0.2595$ & $\mathrm{r}_{148}$ & $=0.2578$ & $\mathrm{r}_{212}$ & $=0.2577$ & $\mathrm{r}_{515}$ & $=0.2581$ \\
\hline$r_{92}$ & $=0.2574$ & $r_{125}$ & $=0.2595$ & $\mathrm{r}_{158}$ & $=0.2578$ & $r_{312}$ & $=0.2577$ & $\mathrm{r}_{615}$ & $=0.2581$ \\
\hline$r_{102}$ & $=0.2574$ & $\mathrm{r}_{135}$ & $=0.2595$ & $\mathrm{r}_{19}$ & $=0.2580$ & $\mathrm{r}_{412}$ & $=0.2577$ & $r_{715}$ & $=0.2581$ \\
\hline$r_{112}$ & $=0.2574$ & $\mathrm{r}_{145}$ & $=0.2595$ & $\mathrm{r}_{29}$ & $=0.2580$ & $\mathrm{r}_{512}$ & $=0.2577$ & $\mathrm{r}_{815}$ & $=0.2581$ \\
\hline $\mathrm{r}_{122}$ & $=0.2574$ & $r_{155}$ & $=0.2595$ & $r_{39}$ & $=0.2580$ & $\mathrm{r}_{612}$ & $=0.2577$ & $\mathrm{r}_{915}$ & $=0.2581$ \\
\hline
\end{tabular}




$\begin{array}{llll}\mathrm{r}_{132}=0.2574 & \mathrm{r}_{16} & =0.2581 \\ \mathrm{r}_{142} & =0.2574 & \mathrm{r}_{26} & =0.2581 \\ \mathrm{r}_{152} & =0.2574 & \mathrm{r}_{36} & =0.2581 \\ \mathrm{r}_{13} & =0.2578 & \mathrm{r}_{46} & =0.2581 \\ \mathrm{r}_{23} & =0.2578 & \mathrm{r}_{56} & =0.2581 \\ \mathrm{r}_{33} & =0.2635 & \mathrm{r}_{66} & =0.2598 \\ \mathrm{r}_{43} & =0.2578 & \mathrm{r}_{76} & =0.2581 \\ \mathrm{r}_{53} & =0.2578 & \mathrm{r}_{86} & =0.2581 \\ \mathrm{r}_{63} & =0.2578 & \mathrm{r}_{96} & =0.2581 \\ \mathrm{r}_{73} & =0.2578 & \mathrm{r}_{106} & =0.2581 \\ \mathrm{r}_{83} & =0.2578 & \mathrm{r}_{116} & =0.2581 \\ \mathrm{r}_{93} & =0.2578 & \mathrm{r}_{126} & =0.2581 \\ \mathrm{r}_{103}=0.2578 & \mathrm{r}_{136} & =0.2581 \\ \mathrm{r}_{113}=0.2578 & \mathrm{r}_{146} & =0.2581 \\ \mathrm{r}_{123}=0.2578 & \mathrm{r}_{156} & =0.2581 \\ \mathrm{r}_{133}=0.2578 & \mathrm{r}_{17} & =0.2578 \\ \mathrm{r}_{143}=0.2578 & \mathrm{r}_{27} & =0.2578 \\ \mathrm{r}_{153}=0.2578 & \mathrm{r}_{37} & =0.2578 \\ \mathrm{r}_{14}=0.2596 & \mathrm{r}_{47} & =0.2578 \\ \mathrm{r}_{24}=0.2596 & \mathrm{r}_{57} & =0.2578 \\ \mathrm{r}_{34}=0.2596 & \mathrm{r}_{67} & =0.2578\end{array}$

$\begin{array}{ll}\mathrm{r}_{49} & =0.2580 \\ \mathrm{r}_{59} & =0.2580 \\ \mathrm{r}_{69} & =0.2580 \\ \mathrm{r}_{79} & =0.2580 \\ \mathrm{r}_{89} & =0.2580 \\ \mathrm{r}_{99} & =0.2617 \\ \mathrm{r}_{109} & =0.2580 \\ \mathrm{r}_{119} & =0.2580 \\ \mathrm{r}_{129} & =0.2580 \\ \mathrm{r}_{139} & =0.2580 \\ \mathrm{r}_{149} & =0.2580 \\ \mathrm{r}_{159} & =0.2580 \\ \mathrm{r}_{110} & =0.2601 \\ \mathrm{r}_{210} & =0.2601 \\ \mathrm{r}_{310} & =0.2601 \\ \mathrm{r}_{410} & =0.2601 \\ \mathrm{r}_{510} & =0.2601 \\ \mathrm{r}_{610} & =0.2601 \\ \mathrm{r}_{710} & =0.2601 \\ \mathrm{r}_{810} & =0.2601 \\ \mathrm{r}_{910} & =0.2601\end{array}$

$\begin{array}{ll}\mathrm{r}_{712} & =0.2577 \\ \mathrm{r}_{812} & =0.2577 \\ \mathrm{r}_{912} & =0.2577 \\ \mathrm{r}_{1012} & =0.2577 \\ \mathrm{r}_{1112} & =0.2577 \\ \mathrm{r}_{1212} & =0.2653 \\ \mathrm{r}_{1312} & =0.2577 \\ \mathrm{r}_{1412} & =0.2577 \\ \mathrm{r}_{1512} & =0.2577 \\ \mathrm{r}_{113} & =0.2578 \\ \mathrm{r}_{213} & =0.2578 \\ \mathrm{r}_{313} & =0.2578 \\ \mathrm{r}_{413} & =0.2578 \\ \mathrm{r}_{513} & =0.2578 \\ \mathrm{r}_{613} & =0.2578 \\ \mathrm{r}_{713} & =0.2578 \\ \mathrm{r}_{813} & =0.2578 \\ \mathrm{r}_{913} & =0.2578 \\ \mathrm{r}_{1013} & =0.2578 \\ \mathrm{r}_{1113} & =0.2578 \\ \mathrm{r}_{1213} & =0.2578\end{array}$

$\begin{array}{ll}r_{1015} & =0.2581 \\ r_{1115} & =0.2581 \\ r_{1215} & =0.2581 \\ r_{1315} & =0.2581 \\ r_{1415} & =0.2581 \\ r_{1515} & =0.2598 \\ r_{116} & =0.1850 \\ r_{216} & =0.1480 \\ r_{316} & =0.0370 \\ r_{416} & =-0.1480 \\ r_{516} & =-0.1110 \\ r_{616} & =0.1300 \\ r_{716} & =0.2590 \\ r_{816} & =0.2040 \\ r_{916} & =0.2220 \\ r_{1016} & =-0.7400 \\ r_{1116} & =0.1670 \\ r_{1216} & =0.2410 \\ r_{1316} & =0.2040 \\ r_{1416} & =0.2040 \\ r_{1516} & =0.1300\end{array}$

The results of that normalized matrix then were able to be converted into matrix-R. The display of the matrix-R can be seen in Figure 2.

$\left[\begin{array}{llllllllllllllll}0.2708 & 0.2574 & 0.2578 & 0.2596 & 0.2595 & 0.2581 & 0.2578 & 0.2578 & 0.2580 & 0.2601 & 0.2580 & 0.2577 & 0.2578 & 0.2578 & 0.2581 & 0.1850 \\ 0.2573 & 0.2690 & 0.2578 & 0.2596 & 0.2595 & 0.2581 & 0.2578 & 0.2578 & 0.2580 & 0.2601 & 0.2580 & 0.2577 & 0.2578 & 0.2578 & 0.2581 & 0.1480 \\ 0.2573 & 0.2574 & 0.2635 & 0.2596 & 0.2595 & 0.2581 & 0.2578 & 0.2578 & 0.2580 & 0.2601 & 0.2580 & 0.2577 & 0.2578 & 0.2578 & 0.2581 & 0.0370 \\ 0.2573 & 0.2574 & 0.2578 & 0.2374 & 0.2595 & 0.2581 & 0.2578 & 0.2578 & 0.2580 & 0.2601 & 0.2580 & 0.2577 & 0.2578 & 0.2578 & 0.2581 & -0.1480 \\ 0.2573 & 0.2574 & 0.2578 & 0.2596 & 0.2393 & 0.2581 & 0.2578 & 0.2578 & 0.2580 & 0.2601 & 0.2580 & 0.2577 & 0.2578 & 0.2578 & 0.2581 & -0.1110 \\ 0.2573 & 0.2574 & 0.2578 & 0.2596 & 0.2595 & 0.2598 & 0.2578 & 0.2578 & 0.2580 & 0.2601 & 0.2580 & 0.2577 & 0.2578 & 0.2578 & 0.2581 & 0.1300 \\ 0.2573 & 0.2574 & 0.2578 & 0.2596 & 0.2595 & 0.2581 & 0.2635 & 0.2578 & 0.2580 & 0.2601 & 0.2580 & 0.2577 & 0.2578 & 0.2578 & 0.2581 & 0.2590 \\ 0.2573 & 0.2574 & 0.2578 & 0.2596 & 0.2595 & 0.2581 & 0.2578 & 0.2635 & 0.2580 & 0.2601 & 0.2580 & 0.2577 & 0.2578 & 0.2578 & 0.2581 & 0.2040 \\ 0.2573 & 0.2574 & 0.2578 & 0.2596 & 0.2595 & 0.2581 & 0.2578 & 0.2578 & 0.2617 & 0.2601 & 0.2580 & 0.2577 & 0.2578 & 0.2578 & 0.2581 & 0.2220 \\ 0.2573 & 0.2574 & 0.2578 & 0.2596 & 0.2595 & 0.2581 & 0.2578 & 0.2578 & 0.2580 & 0.2299 & 0.2580 & 0.2577 & 0.2578 & 0.2578 & 0.2581 & -0.7400 \\ 0.2573 & 0.2574 & 0.2578 & 0.2596 & 0.2595 & 0.2581 & 0.2578 & 0.2578 & 0.2580 & 0.2601 & 0.2617 & 0.2577 & 0.2578 & 0.2578 & 0.2581 & 0.1670 \\ 0.2573 & 0.2574 & 0.2578 & 0.2596 & 0.2595 & 0.2581 & 0.2578 & 0.2578 & 0.2580 & 0.2601 & 0.2580 & 0.2653 & 0.2578 & 0.2578 & 0.2581 & 0.2410 \\ 0.2573 & 0.2574 & 0.2578 & 0.2596 & 0.2595 & 0.2581 & 0.2578 & 0.2578 & 0.2580 & 0.2601 & 0.2580 & 0.2577 & 0.2635 & 0.2578 & 0.2581 & 0.2040 \\ 0.2573 & 0.2574 & 0.2578 & 0.2596 & 0.2595 & 0.2581 & 0.2578 & 0.2578 & 0.2580 & 0.2601 & 0.2580 & 0.2577 & 0.2578 & 0.2635 & 0.2581 & 0.2040 \\ 0.2573 & 0.2574 & 0.2578 & 0.2596 & 0.2595 & 0.2581 & 0.2578 & 0.2578 & 0.2580 & 0.2601 & 0.2580 & 0.2577 & 0.2578 & 0.2578 & 0.2598 & 0.1300\end{array}\right]$

Figure 2. Matrix-R.

Based on the values of matrix-R and Equation 3, then the calculations were able to be performed to determine the matrix-Y. The display of matrix-Y can be seen in Figure 3.

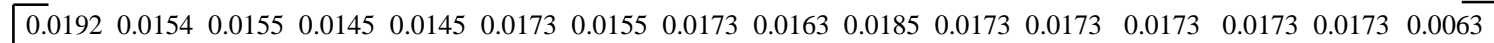
$\begin{array}{lllllllllllllllll}0.0183 & 0.0161 & 0.0155 & 0.0145 & 0.0145 & 0.0173 & 0.0155 & 0.0173 & 0.0163 & 0.0185 & 0.0173 & 0.0173 & 0.0173 & 0.0173 & 0.0173 & 0.0050\end{array}$ $\begin{array}{lllllllllllllllll}0.0183 & 0.0154 & 0.0158 & 0.0145 & 0.0145 & 0.0173 & 0.0155 & 0.0173 & 0.0163 & 0.0185 & 0.0173 & 0.0173 & 0.0173 & 0.0173 & 0.0173 & 0.0013\end{array}$ $\begin{array}{llllllllllllllll}0.0183 & 0.0154 & 0.0155 & 0.0133 & 0.0145 & 0.0173 & 0.0155 & 0.0173 & 0.0163 & 0.0185 & 0.0173 & 0.0173 & 0.0173 & 0.0173 & 0.0173 & -0.0050\end{array}$ $\begin{array}{lllllllllllllllll}0.0183 & 0.0154 & 0.0155 & 0.0145 & 0.0134 & 0.0173 & 0.0155 & 0.0173 & 0.0163 & 0.0185 & 0.0173 & 0.0173 & 0.0173 & 0.0173 & 0.0173 & -0.0038\end{array}$ $\begin{array}{lllllllllllllllll}0.0183 & 0.0154 & 0.0155 & 0.0145 & 0.0145 & 0.0174 & 0.0155 & 0.0173 & 0.0163 & 0.0185 & 0.0173 & 0.0173 & 0.0173 & 0.0173 & 0.0173 & 0.0044\end{array}$ $\begin{array}{llllllllllllllll}0.0183 & 0.0154 & 0.0155 & 0.0145 & 0.0145 & 0.0173 & 0.0158 & 0.0173 & 0.0163 & 0.0185 & 0.0173 & 0.0173 & 0.0173 & 0.0173 & 0.0173 & 0.0088\end{array}$ $\begin{array}{llllllllllllllllll}0.0183 & 0.0154 & 0.0155 & 0.0145 & 0.0145 & 0.0173 & 0.0155 & 0.0177 & 0.0163 & 0.0185 & 0.0173 & 0.0173 & 0.0173 & 0.0173 & 0.0173 & 0.0069\end{array}$ $\begin{array}{lllllllllllllllll}0.0183 & 0.0154 & 0.0155 & 0.0145 & 0.0145 & 0.0173 & 0.0155 & 0.0173 & 0.0165 & 0.0185 & 0.0173 & 0.0173 & 0.0173 & 0.0173 & 0.0173 & 0.0075\end{array}$ $\begin{array}{llllllllllllllll}0.0183 & 0.0154 & 0.0155 & 0.0145 & 0.0145 & 0.0173 & 0.0155 & 0.0173 & 0.0163 & 0.0163 & 0.0173 & 0.0173 & 0.0173 & 0.0173 & 0.0173 & -0.0252\end{array}$ $\begin{array}{llllllllllllllll}0.0183 & 0.0154 & 0.0155 & 0.0145 & 0.0145 & 0.0173 & 0.0155 & 0.0173 & 0.0163 & 0.0185 & 0.0175 & 0.0173 & 0.0173 & 0.0173 & 0.0173 & 0.0057\end{array}$ $\begin{array}{lllllllllllllllll}0.0183 & 0.0154 & 0.0155 & 0.0145 & 0.0145 & 0.0173 & 0.0155 & 0.0173 & 0.0163 & 0.0185 & 0.0173 & 0.0178 & 0.0173 & 0.0173 & 0.0173 & 0.0082\end{array}$ $\begin{array}{lllllllllllllllll}0.0183 & 0.0154 & 0.0155 & 0.0145 & 0.0145 & 0.0173 & 0.0155 & 0.0173 & 0.0163 & 0.0185 & 0.0173 & 0.0173 & 0.0177 & 0.0173 & 0.0173 & 0.0069\end{array}$ $\begin{array}{llllllllllllllll}0.0183 & 0.0154 & 0.0155 & 0.0145 & 0.0145 & 0.0173 & 0.0155 & 0.0173 & 0.0163 & 0.0185 & 0.0173 & 0.0173 & 0.0173 & 0.0177 & 0.0173 & 0.0069\end{array}$ $\begin{array}{llllllllllllllll}0.0183 & 0.0154 & 0.0155 & 0.0145 & 0.0145 & 0.0173 & 0.0155 & 0.0173 & 0.0163 & 0.0185 & 0.0173 & 0.0173 & 0.0173 & 0.0173 & 0.0174 & 0.0044\end{array}$

Figure 3. Matrix-Y.

After the matrix-Y was obtained, so the matrix of negative ideal solutions was able to be calculated used Equation 4, and the matrix of positive ideal solutions used Equation 5 with assuming all evaluation aspects were included in the 'profit attribute'. The calculation results of the positive and negative ideal solution matrixes can be seen as follows. 
a) matrix of negative ideal solutions

$\mathrm{A}^{-}=\{0.0183 ; 0.0154 ; 0.0155 ; 0.0133 ; 0.0134 ; 0.0173 ; 0.0155 ; 0.0173 ; 0.0163 ; 0.0163 ; 0.0173 ; 0.0173 ; 0.0173 ;$ $0.0173 ; 0.0173 ;-0.0252\}$

b) matrix of positive ideal solutions

$\mathrm{A}^{+}=\{0.0192 ; 0.0161 ; 0.0158 ; 0.0145 ; 0.0145 ; 0.0174 ; 0.0158 ; 0.0177 ; 0.0165 ; 0.0185 ; 0.0175 ; 0.0178 ; 0.0177 ;$ $0.0177 ; 0.0174 ; 0.0088\}$

After the matrix value of the negative ideal solutions and positive ideal solutions were obtained, then the distance between the values of each indicator was calculated with the negative ideal solutions that used Equation 6 and positive ideal solutions that used Equation 7. The calculation results intended can be seen as follows.

a) Calculation results of the distance between the values of each indicator with the negative ideal solutions matrix;

$$
\begin{aligned}
& \mathrm{D}_{1}{ }^{-}=0.0316 ; \mathrm{D}_{2}{ }^{-}=0.0303 ; \mathrm{D}_{3}{ }^{-}=0.0266 ; \mathrm{D}_{4}{ }^{-}=0.0203 ; \mathrm{D}_{5}{ }^{-}=0.0215 ; \mathrm{D}_{6}{ }^{-}=0.0297 ; \mathrm{D}_{7}{ }^{-}=0.0341 ; \mathrm{D}_{8}{ }^{-}=0.0322 ; \\
& \mathrm{D}_{9}{ }^{-}=0.0328 ; \mathrm{D}_{10^{-}}=0.0017 ; \mathrm{D}_{11^{-}}=0.0310 ; \mathrm{D}_{12}=0.0335 ; \mathrm{D}_{13}{ }^{-}=0.0322 ; \mathrm{D}_{14^{-}}=0.0322 ; \mathrm{D}_{15}=0.0297 .
\end{aligned}
$$

b) Calculation results of the distance between the values of each indicator with the positive ideal solutions matrix;

$\mathrm{D}_{1}{ }^{+}=0.0028 ; \mathrm{D}_{2}{ }^{+}=0.0040 ; \mathrm{D}_{3}{ }^{+}=0.0077 ; \mathrm{D}_{4}{ }^{+}=0.0140 ; \mathrm{D}_{5}{ }^{+}=0.0127 ; \mathrm{D}_{6}{ }^{+}=0.0047 ; \mathrm{D}_{7}{ }^{+}=0.0015 ; \mathrm{D}_{8}{ }^{+}=0.0024 ;$

$\mathrm{D}_{9}{ }^{+}=0.0020 ; \mathrm{D}_{10}{ }^{+}=0.0341 ; \mathrm{D}_{11}{ }^{+}=0.0035 ; \mathrm{D}_{12}{ }^{+}=0.0016 ; \mathrm{D}_{13}{ }^{+}=0.0024 ; \mathrm{D}_{14}{ }^{+}=0.0024 ; \mathrm{D}_{15}{ }^{+}=0.0047$.

After the calculation results of the negative and positive ideal solutions matrix were obtained, so was able to be performed the calculations of preference scores for each indicator. The calculation results intended can be seen as follows.

$\mathrm{V}_{1}=0.918 ; \mathrm{V}_{2}=0.883 ; \mathrm{V}_{3}=0.775 ; \mathrm{V}_{4}=0.592 ; \mathrm{V}_{5}=0.628 ; \mathrm{V}_{6}=0.864 ; \mathrm{V}_{7}=0.957 ; \mathrm{V}_{8}=0.930 ; \mathrm{V}_{9}=0.943 ; \mathrm{V}_{10}=$ $0.047 ; \mathrm{V}_{11}=0.899 ; \mathrm{V}_{12}=0.954 ; \mathrm{V}_{13}=0.930 ; \mathrm{V}_{14}=0.930 ; \mathrm{V}_{15}=0.864$

The preference scores for each indicator were recapitulated entirely in the form of bar charts. The display of bar charts intended can be seen in Figure 4.

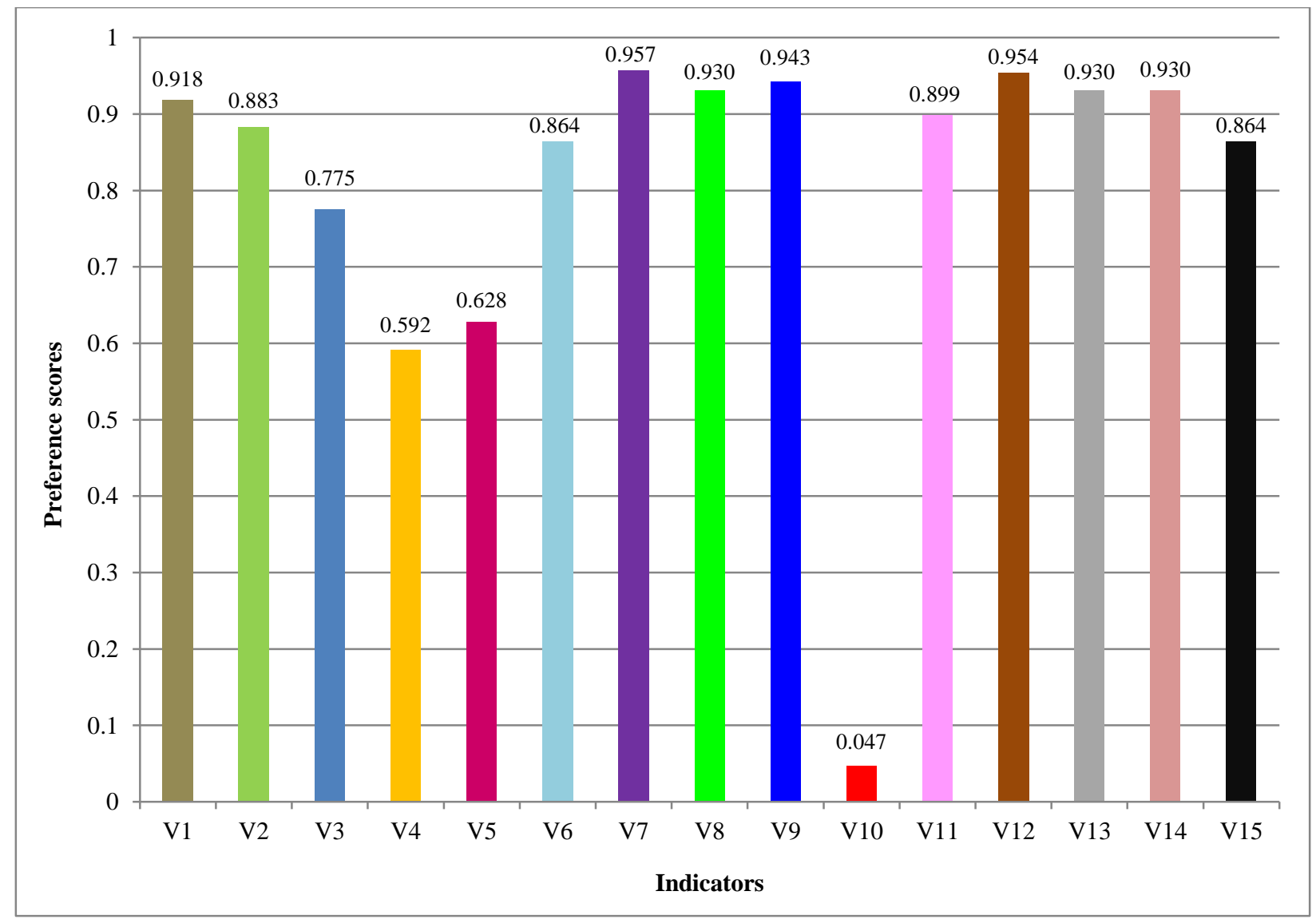

Figure 4. Recapitulation of preference scores for each evaluation indicator.

Based on the diagram shown in Figure 4, it was able to be stated that the aspect that becomes the priority of improvement to realize the effectiveness of blended learning implementation was the aspect of "fund management". 
This statement was evidenced by the I-10 indicator that gets the lowest preference score $\left(\mathrm{V}_{10}=0.047\right)$ when compared to other indicators. A simulation trial of the TOPSIS calculation was carried out to determine the effectiveness percentage of the TOPSIS calculation process. The results of a simulation trial that were conducted by 30 respondents can be seen in Table 7 .

Table 7. Trial results of TOPSIS calculation to determine the dominant aspect that priority for improvement.

\begin{tabular}{|c|c|c|c|c|c|c|c|c|c|c|c|c|c|}
\hline \multirow{2}{*}{ No. } & \multirow{2}{*}{ Respondents } & \multicolumn{10}{|c|}{ Items- } & \multirow{2}{*}{$\Sigma$} & \multirow{2}{*}{$\begin{array}{c}\text { Effectiveness } \\
\text { Percentage (\%) }\end{array}$} \\
\hline & & 1 & 2 & 3 & 4 & 5 & 6 & 7 & 8 & 9 & 10 & & \\
\hline 1 & Teacher-1 & 4 & 5 & 4 & 5 & 4 & 4 & 5 & 4 & 5 & 5 & 45 & 90.00 \\
\hline 2 & Teacher-2 & 4 & 4 & 4 & 5 & 4 & 4 & 5 & 4 & 4 & 4 & 42 & 84.00 \\
\hline 3 & Teacher-3 & 5 & 4 & 4 & 4 & 4 & 4 & 4 & 4 & 4 & 4 & 41 & 82.00 \\
\hline 4 & Teacher-4 & 4 & 4 & 4 & 4 & 5 & 5 & 5 & 4 & 5 & 5 & 45 & 90.00 \\
\hline 5 & Teacher-5 & 4 & 5 & 4 & 5 & 4 & 5 & 4 & 5 & 4 & 5 & 45 & 90.00 \\
\hline 6 & Teacher-6 & 4 & 4 & 4 & 4 & 5 & 4 & 5 & 4 & 5 & 4 & 43 & 86.00 \\
\hline 7 & Teacher-7 & 5 & 4 & 4 & 5 & 4 & 4 & 4 & 5 & 4 & 4 & 43 & 86.00 \\
\hline 8 & Teacher-8 & 4 & 4 & 4 & 4 & 5 & 4 & 4 & 4 & 5 & 4 & 42 & 84.00 \\
\hline 9 & Teacher-9 & 5 & 4 & 4 & 5 & 4 & 5 & 4 & 5 & 4 & 5 & 45 & 90.00 \\
\hline 10 & Teacher-10 & 4 & 4 & 4 & 4 & 5 & 4 & 5 & 5 & 5 & 4 & 44 & 88.00 \\
\hline 11 & Teacher-11 & 5 & 4 & 4 & 4 & 4 & 4 & 4 & 4 & 4 & 5 & 42 & 84.00 \\
\hline 12 & Teacher-12 & 4 & 5 & 5 & 4 & 5 & 4 & 5 & 4 & 5 & 4 & 45 & 90.00 \\
\hline 13 & Teacher-13 & 5 & 4 & 4 & 5 & 5 & 4 & 4 & 5 & 4 & 5 & 45 & 90.00 \\
\hline 14 & Teacher-14 & 4 & 4 & 4 & 4 & 5 & 4 & 5 & 4 & 4 & 4 & 42 & 84.00 \\
\hline 15 & Teacher-15 & 5 & 4 & 5 & 4 & 4 & 4 & 5 & 4 & 4 & 5 & 44 & 88.00 \\
\hline 16 & Teacher-16 & 4 & 5 & 4 & 4 & 5 & 5 & 5 & 4 & 5 & 5 & 46 & 92.00 \\
\hline 17 & Teacher-17 & 5 & 4 & 5 & 5 & 4 & 4 & 4 & 5 & 4 & 4 & 44 & 88.00 \\
\hline 18 & Teacher-18 & 4 & 5 & 4 & 4 & 5 & 4 & 5 & 4 & 5 & 4 & 44 & 88.00 \\
\hline 19 & Teacher-19 & 5 & 4 & 5 & 4 & 4 & 5 & 4 & 5 & 4 & 5 & 45 & 90.00 \\
\hline 20 & Teacher-20 & 5 & 5 & 4 & 4 & 5 & 4 & 5 & 4 & 5 & 5 & 46 & 92.00 \\
\hline 21 & Teacher-21 & 5 & 4 & 4 & 4 & 5 & 4 & 4 & 5 & 4 & 5 & 44 & 88.00 \\
\hline 22 & Teacher-22 & 4 & 5 & 4 & 4 & 5 & 4 & 5 & 4 & 4 & 4 & 43 & 86.00 \\
\hline 23 & Teacher-23 & 5 & 4 & 5 & 4 & 4 & 4 & 5 & 4 & 4 & 5 & 44 & 88.00 \\
\hline 24 & Teacher-24 & 4 & 5 & 4 & 4 & 4 & 4 & 5 & 4 & 5 & 5 & 44 & 88.00 \\
\hline 25 & Teacher-25 & 5 & 4 & 4 & 5 & 4 & 4 & 4 & 5 & 4 & 5 & 44 & 88.00 \\
\hline 26 & Teacher-26 & 4 & 4 & 4 & 4 & 5 & 4 & 5 & 4 & 5 & 4 & 43 & 86.00 \\
\hline 27 & Teacher-27 & 4 & 4 & 5 & 5 & 4 & 4 & 4 & 5 & 4 & 4 & 43 & 86.00 \\
\hline 28 & Teacher-28 & 4 & 4 & 4 & 4 & 5 & 4 & 5 & 4 & 5 & 5 & 44 & 88.00 \\
\hline 29 & Teacher-29 & 5 & 4 & 5 & 5 & 4 & 5 & 4 & 5 & 4 & 4 & 45 & 90.00 \\
\hline 30 & Teacher-30 & 5 & 4 & 4 & 4 & 4 & 4 & 5 & 4 & 4 & 5 & 43 & 86.00 \\
\hline & & & & & & & & & & & & & 87.67 \\
\hline
\end{tabular}

Notes:

Item-1: Initial data completeness for normalized matrix calculations;

Item-2: Accuracy of the normalized matrix calculation process;

Item-3: Accuracy of normalized data matrix conversion into matrix-R;

Item-4: The accuracy of the calculation process for matrix-R data becomes matrix-Y data;

Item-5: The calculation process accuracy of the negative ideal solutions matrix;

Item-6: The calculation process accuracy of the positive ideal solutions matrix;

Item-7: The accuracy of the calculation process the distance between the values of each indicator with the negative ideal solutions matrix;

Item-8: The accuracy of the calculation process the distance between the values of each indicator with the positive ideal solutions matrix;

Item-9: Accuracy in the calculation process the preference scores;

Item-10: The accuracy of decision making based on each indicator's preference scores. 
Based on the initial trial results on the Tat Twam Asi-Discrepancy evaluation model based on TOPSIS previously shown in Table 5, it was able to be stated that the evaluation model design was categorized as good quality. This was supported by the results of the test that showed a quality percentage was $87.00 \%$. If viewed from the design quality standards that refer to the eleven's scale, then the quality percentage was $87.00 \%$, so included in a good category with scores range of $85-94 \%$. Therefore, there is no need to make major revisions to the design of the evaluation model, and the design is ready to be used for the next development stage.

Based on the trial results of a TOPSIS calculation simulation that had shown previously in Table 7, it was able to be stated that the TOPSIS calculation had been effectively was used to determine the dominant aspect that needs to be improved. This was evidenced by the simulation trial results, which showed the effectiveness percentage was $87.67 \%$. If viewed from the calculation effectiveness standards that refer to the five's scale, then the quality percentage was $87.67 \%$, so included in an effective category with scores range of $80-89 \%$. Therefore, there is no need to retest the TOPSIS calculation simulation. TOPSIS method is accurate and ready to be applied at the next development stage.

This research had been able to answer the problems found in previous studies. The limitations of Mutawa (2017), Mantara et al. (2021), and Habib and Ramzan (2020) $[11,15,16]$ had been answered through this research by showing that there were aspects of Discrepancy evaluation were used as a reference for evaluating the blended learning implementation at IT vocational schools in Bali. Limitations of Lippe and Carter (2018), Martín-Martínez et al. (2020), Naibaho (2021) $[12,14,17]$ had been answered through this research by showing the TOPSIS calculation process in determining the dominant aspects that must be improved. The limitation of Thurab-Nkhosi (2019) [13] had been answered through this research by presenting the results of a complete and comprehensive evaluation of the defining, installation, process, and product components. That presentation of the evaluation results can be seen in Table 3 in the column section of "Percentage of Assessment from 30 Respondents". Besides those advantages, this research also had an obstacle. The obstacle was the TOPSIS calculation was still done manually and was not applied to a computer application, so the speed of the calculation results was not optimal.

\section{4- Conclusion}

The results of the combination of the Discrepancy evaluation model, the Tat Twam Asi concept, and the TOPSIS method had presented innovations in the field of educational evaluation in the form of a design of the Tat Twam AsiDiscrepancy evaluation model based on TOPSIS. That evaluation model design had been successfully made and had passed a series of trials in this research. Based on a series of trial results that was conducted on the evaluation model design, it was stated that the design of the Tat Twam Asi-Discrepancy evaluation model based on TOPSIS was feasible to be used to determine the dominant aspects that needed to be improved to realize the effectiveness of blended learning implementation (especially at IT vocational schools in Bali). The feasibility of using the evaluation model design was evidenced by the quality percentage average was $87.00 \%$ obtained from the preliminary trials toward the design of the Tat Twam Asi-Discrepancy evaluation model based on TOPSIS. That percentage indicates the evaluation model design was very good quality if viewed from quality standards of eleven's scale. In addition, the feasibility of using the evaluation model design was also evidenced from the effectiveness percentage average was $87.67 \%$ obtained from the trials of TOPSIS calculation simulation to determine the dominant aspects which were improvement priority. This percentage also indicates that the evaluation model was included in the effective category based on the effectiveness standards of the five's scale. The results of this research have a positive impact on the field of educational evaluation because this research shows innovations in determining the most dominant aspects for improvement so that the evaluation recommendations will be more precise. The contribution of the results of this research shows the design of an evaluation model that can be integrated with other evaluation models to make it easier to determine the most dominant aspects for improvement so the program being evaluated becomes more enhance. Future work to be done is to develop this evaluation model towards desktop-based or web-based applications so that access and process of evaluation calculation can be carried out quickly and accurately.

\section{5- Declarations}

\section{5-1-Author Contributions}

Conceptualization, D.G.H.D.; methodology, D.G.H.D.; formal analysis, D.G.H.D., A.A., and P.W.A.S.; investigation, D.G.H.D.; data curation, D.G.H.D., A.A., and P.W.A.S.; writing — original draft preparation, D.G.H.D.; writing - review and editing, D.G.H.D. All authors have read and agreed to the published version of the manuscript.

\section{5-2-Data Availability Statement}

The data presented in this study are available in article.

\section{5-3-Funding}

The authors received financial support from the Directorate General of Research and Development, Ministry of Education, Culture, Research and Technology of the Republic of Indonesia for the research, authorship, and/or publication of this article. 


\section{5-4-Acknowledgements}

The authors also express their gratitude to the Chancellor and Chair of the Research and Community Service Institute, Universitas Pendidikan Ganesha, who give permission and opportunity to the authors for carrying out this research.

\section{5-5-Conflicts of Interest}

The authors declare that there is no conflict of interests regarding the publication of this manuscript. In addition, the ethical issues, including plagiarism, informed consent, misconduct, data fabrication and/or falsification, double publication and/or submission, and redundancies have been completely observed by the authors.

\section{6- References}

[1] Irawan, Vincentius Tjandra, Eddy Sutadji, and Widiyanti. "Blended Learning Based on Schoology: Effort of Improvement Learning Outcome and Practicum Chance in Vocational High School." Edited by Hau Fai Edmond Law. Cogent Education 4, no. 1 (January 1, 2017): 1-10. doi:10.1080/2331186x.2017.1282031.

[2] Suyasa, P Wayan Arta, Putu Sukma Kurniawan, I Putu Wisna Ariawan, Wayan Sugandini, Ni Desak Made Sri Adnyawati, I Dewa Ayu Made Budhyani, and Dewa Gede Hendra Divayana. "Empowerment of CSE-UCLA Model Based on Glickman Quadrant Aided by Visual Application to Evaluate the Blended Learning Program on SMA Negeri 1 Ubud." Journal of Theoretical and Applied Information Technology 96, no. 18 (2018), 6203-6219.

[3] Savoie-Roskos, Mateja R., Stacy Bevan, Rebecca Charlton, and Marlene Israelsen Graf. "Approaches to Evaluating Blended Courses.” Journal on Empowering Teaching Excellence 2, no. 1 (2018): 3-11. doi:10.26077/jg9k-5e05.

[4] Pima, John Marco, Michael Odetayo, Rahat Iqbal, and Eliamani Sedoyeka. "A Thematic Review of Blended Learning in Higher Education." International Journal of Mobile and Blended Learning 10, no. 1 (January 2018): 1-11. doi:10.4018/ijmbl.2018010101.

[5] Jayanta, I Nyoman Laba, Kadek Dewi Suryantari, and Made Sumantri. "An Analysis of Discrepancy between the Lesson Plan and the Implementation of Curriculum 2013 in Teaching and Learning Process in SD Negeri 4 Kaliuntu." Journal of Education Research and Evaluation 1, no. 2 (May 2, 2017): 73-81. doi:10.23887/jere.v1i2.9839.

[6] Said, Meldasari, R. Madhakomala, and Fahmi Idris. "Discrepancy Evaluation Model for Human Resources Health Placement Evaluation at the Puskesmas.” Journal of Environmental Treatment Techniques 7, no. 4 (2019): 588-594.

[7] Sanjaya, Dewa Bagus. "Reconstructing Local Wisdom Based Character Education for Sekaa Teruna Teruni in Desa Pakraman Ubud Bali.” International Research Journal of Management, IT \& Social Sciences 4, no. 2 (2017): 190-197.

[8] Perbowosari, Heny. "The Local Wisdom Value of Mandhasiya Tradition (Study of Hindu Education)." Vidyottama Sanatana: International Journal of Hindu Science and Religious Studies 3, no. 1 (May 1, 2019): 1-12. doi:10.25078/ijhsrs.v3i1.790.

[9] Krohling, Renato A., and André G.C. Pacheco. "A-TOPSIS - An Approach Based on TOPSIS for Ranking Evolutionary Algorithms.” Procedia Computer Science 55 (2015): 308-317. doi:10.1016/j.procs.2015.07.054.

[10] Song, Jing, and Junhui Zheng. "The Application of Grey-TOPSIS Method on Teaching Quality Evaluation of the Higher Education." International Journal of Emerging Technologies in Learning (IJET) 10, no. 8 (December 14, 2015): 42-45. doi:10.3991/ijet.v10i8.5219.

[11] Mutawa, A.M. "Evaluation of Blended Learning in Higher Education: A Case Study." PEOPLE: International Journal of Social Sciences 3, no. 1 (January 1, 2017): 881-889. doi:10.20319/pijss.2017.s31.881889.

[12] Lippe, Megan, and Patricia Carter. "Using the CIPP Model to Assess Nursing Education Program Quality and Merit." Teaching and Learning in Nursing 13, no. 1 (January 2018): 9-13. doi:10.1016/j.teln.2017.09.008.

[13] Thurab-Nkhosi, D. "The Evaluation of a Blended Faculty Development Course Using the CIPP Framework." International Journal of Education and Development using Information and Communication Technology 15, no. 1, (2019): $245-254$.

[14] Martín-Martínez, Laura, Vanesa Sainz, and Fidel Rodríguez-Legendre. "Evaluation of a Blended Learning Model for PreService Teachers.” Knowledge Management \& E-Learning 12, no. 2, (June 2020): 147-164. doi:10.34105/j.kmel.2020.12.008.

[15] Mantara, Angga Yuni, Endang Prastuti, and Titis Setyo Wahyudi. "Student's Evaluation on the Use of Learning Media during Pandemic.” KnE Social Sciences (January 5, 2021): 446-453. doi:10.18502/kss.v4i15.8232.

[16] Habib, Mohamed, and Muhammad Ramzan. "Performance Assessment and Analysis of Blended Learning in IT Education: A Longitudinal Study in Saudi Electronic University.” International Journal of Advanced Computer Science and Applications 11, no. 6 (2020): 446-453. doi:10.14569/ijacsa.2020.0110610.

[17] Naibaho, Lamhot. "Online Learning Evaluation during Covid-19 Using CSE-UCLA Evaluation Model at English Education Department Universitas Kristen Indonesia." Budapest International Research and Critics Institute (BIRCI-Journal): Humanities and Social Sciences 4, no. 2 (April 28, 2021): 1987-1997. doi:10.33258/birci.v4i2.1887. 
[18] Agung, Anak Agung Gede, I Gusti Putu Sudiarta, and Dewa Gede Hendra Divayana. "The Quality Evaluation of School Management Model Based on Balinese Local Wisdom Using Weighted Product Calculation." Journal of Theoretical and Applied Information Technology 96, no. 19, (2018), 6570-6579.

[19] Sugiharni, Gusti Ayu Dessy. "The Development of Interactive Instructional Media Oriented to Creative Problem Solving Model on Function Graphic Subject.” Journal of Education Research and Evaluation 2, no. 4 (February 4, 2019): $183-189$. doi:10.23887/jere.v2i4.16694.

[20] Fikri, H., Madona, A.S., and Morelent, Y. "The Practicality and Effectiveness of Interactive Multimedia in Indonesian Language Learning at the 5th Grade of Elementary School.” The Journal of Social Sciences Research no. SPI 2 (November 15, 2018): 531-539. doi:10.32861/jssr.spi2.531.539.

[21] Maryansyah, Yupika. "An Analysis on Readability of English Reading Texts for Grade IX Students at MTsN 2 Kota Bengkulu.” Premise Journal 5, no. 1 (April 30, 2016): 69-88. doi:10.24127/pj.v5i1.416.

[22] Panda, Monalisa, and Alok Kumar Jagadev. “TOPSIS in Multi-Criteria Decision Making: A Survey.” $20182^{\text {nd }}$ International Conference on Data Science and Business Analytics (ICDSBA) (September 2018): 51-54. doi:10.1109/icdsba.2018.00017.

[23] Kaur, Simarpreet, Sumeet Kaur Sehra, and Sukhijt Singh Sehra. "A Framework for Software Quality Model Selection Using TOPSIS.” 2016 IEEE International Conference on Recent Trends in Electronics, Information \& Communication Technology (RTEICT) (May 2016): 736-739. doi:10.1109/rteict.2016.7807922.

[24] Bandyopadhyay, Susmita. “Application of Fuzzy Probabilistic TOPSIS on a Multi-Criteria Decision Making Problem.” 2017 Second International Conference on Electrical, Computer and Communication Technologies (ICECCT) (February 2017): 1-3. doi:10.1109/icecct.2017.8118038.

[25] Zhang, Ling, Yan Xu, Chung-Hsing Yeh, Le He, and De-Qun Zhou. "Bi-TOPSIS: A New Multi-criteria Decision Making Method for Interrelated Criteria with Bipolar Measurement." IEEE Transactions on Systems, Man, and Cybernetics: Systems 47, no. 12 (December 2017): 3272-3283. doi:10.1109/tsmc.2016.2573582.

[26] Baylan, Emin Başar. "A Novel Project Risk Assessment Method Development via AHP-TOPSIS Hybrid Algorithm." Emerging Science Journal 4, no. 5 (October 1, 2020): 390-410. doi:10.28991/esj-2020-01239.

[27] Zheng, Feng, and Yang-Cheng Lin. "A Fuzzy TOPSIS Expert System Based on Neural Networks for New Product Design.” 2017 International Conference on Applied System Innovation (ICASI), Sapporo, Japan (May 2017): 598-601. doi:10.1109/icasi.2017.7988494. 